\title{
Management of Full-Thickness Macular Hole in A Genetically Confirmed Case with Usher Syndrome
}

\author{
Evangelia S. Panagiotou - Thomas Papathomas - Konstantinos Nikopoulos • \\ Stavrenia Koukoula • Mathieu Quinodoz • Atta Ur Rehman • \\ Theodoros Giannopoulos · Carlo Rivolta · Anastasios G. Konstas
}

Received: May 12, 2020 / Published online: June 21, 2020

(C) The Author(s) 2020

\begin{abstract}
Introduction: Full-thickness macular hole (FTMH) formation is rarely seen in patients with retinitis pigmentosa (RP) and can have an adverse impact on their residual visual function. The underlying mechanisms are unknown, and clinical experience is limited regarding surgical outcomes. Here, we describe the surgical management of FTMH in a young patient with genetically confirmed Usher syndrome, the most common form of syndromic RP.

Case Report: A 28-year-old woman presented with blurred vision in her right eye (RE). She
\end{abstract}

Digital Features To view digital features for this article go to https://doi.org/10.6084/m9.figshare.12459272

E. S. Panagiotou - T. Papathomas - T. Giannopoulos - A. G. Konstas $(\bowtie)$

1st Department of Ophthalmology, Aristotle

University of Thessaloniki, AHEPA Hospital,

Thessaloniki, Greece

e-mail: agkonstas@gmail.com

K. Nikopoulos

Laboratory of Oncogenomics, Department of

Hematology, Lausanne University Hospital (CHUV),

Lausanne, Switzerland

S. Koukoula

Ophthalmica Institute, Thessaloniki, Greece

M. Quinodoz · C. Rivolta

Department of Genetics and Genome Biology,

University of Leicester, Leicester, UK had a history of RP and bilateral hearing impairment since childhood. Fundoscopy and spectral-domain optical coherence tomography revealed a FTMH in the RE along with typical RP features bilaterally. After pars plana vitrectomy (PPV) with internal limiting membrane peel and gas tamponade, the FTMH closed. Six months after PPV the patient underwent cataract surgery in the affected eye, and the visual acuity remained stable compared to baseline. The clinical diagnosis of Usher syndrome was genetically confirmed by whole exome sequencing (WES), which revealed the presence of two pathogenic nucleotide variants in trans (compound heterozygosity) in the gene USH2A. Conclusion: We report a rare case of successful closure of a FTMH in a patient with Usher

M. Quinodoz · C. Rivolta Institute of Molecular and Clinical Ophthalmology Basel (IOB), Basel, Switzerland

M. Quinodoz · C. Rivolta Department of Ophthalmology, University of Basel, Basel, Switzerland

A. U. Rehman

Division of Genetic Medicine, Lausanne University Hospital and University of Lausanne, Lausanne, Switzerland

A. G. Konstas

3rd Department of Ophthalmology, Aristotle University of Thessaloniki, AHEPA Hospital,

Thessaloniki, Greece 
syndrome. Surgical treatment of FTMH can help preserve the central vision in RP patients, whose peripheral vision is severely affected.

Keywords: Full-thickness macular hole; Pars plana vitrectomy; Retinitis pigmentosa; Usher syndrome

\section{Key Summary Points}

Full-thickness macular hole (FTMH) formation is rarely seen in patients with retinitis pigmentosa (RP) and can have an adverse impact on their residual visual function, while clinical experience is limited regarding surgical outcomes.

We report a rare case of successful closure of a FTMH in a patient with Usher syndrome, the most common form of syndromic RP, which was genetically confirmed.

This is the first case of FTMH in Usher syndrome reporting surgical outcomes combined with genetic data.

Vitrectomy appears to be effective in these cases and should be performed to try to preserve the central vision of RP patients who usually have severely impaired peripheral vision.

\section{INTRODUCTION}

Usher syndrome is an autosomal recessive disorder characterised by retinitis pigmentosa (RP), sensorineural hearing loss and in some cases vestibular dysfunction [1] with a prevalence estimated to be between 6.2 and $16.67 / 100,000$ $[2,3]$. It is the most common form of syndromic RP and accounts for about 10\% of inherited blindness caused by retinal dystrophies and optic neuropathies [4]. Usher syndrome is genetically heterogeneous and to date more than ten genes have been identified as causative of the disease $[5,6]$.
Although in RP the peripheral visual field is typically constricted with initial sparing of the central retina, macular abnormalities are often observed, leading to reduction of the visual acuity (VA). Cystoid macular oedema and epiretinal membrane are the most common macular anomalies in these patients, while the presence of a macular hole is rare $[7,8]$. Since in RP patients the peripheral vision is affected, it is important to detect and treat any potential macular abnormalities to preserve central vision. Due to the limited experience in cases of macular hole in RP, it is useful to study the outcomes and prognosis of surgical treatment. Here, we report the anatomical and functional outcomes of surgical management in a young patient with Usher syndrome and full-thickness macular hole (FTMH). The genetic pathogenic variants underlying the patient's condition are also presented.

\section{CASE PRESENTATION}

A 28-year-old woman was referred to our department with a complaint of blurred vision during a period of 1 month in her right eye (RE). She reported nyctalopia and restricted visual field in both eyes since childhood and had been diagnosed RP with no family history. She also had had bilateral hearing impairment since childhood and wore hearing aids.

At presentation, her best-corrected VA was $2.5 / 10(-1.00 \mathrm{sph})$ in the $\mathrm{RE}$ and $3.5 / 10$ ($\left.1.00 \mathrm{cyl} \times 90^{\circ}\right)$ in the left eye (Snellen). Anterior segment examination was unremarkable with bilateral clear lenses. Dilated fundoscopy revealed a full-thickness macular hole and posterior vitreous detachment in the RE. In addition, both eyes showed typical signs of RP including peripheral retinal atrophy with bone spicule-shaped pigmentation in the mid periphery, waxy pallor of the optic nerve head and attenuation of retinal vessels (Fig. 1). Spectral domain optical coherence tomography (SDOCT) confirmed the presence of a FTMH stage IV in the RE along with atrophy of the outer retinal layers and absence of the ellipsoid zone (Fig. 2a). In the fellow eye, thinning of the outer retinal layers was also observed, while the 

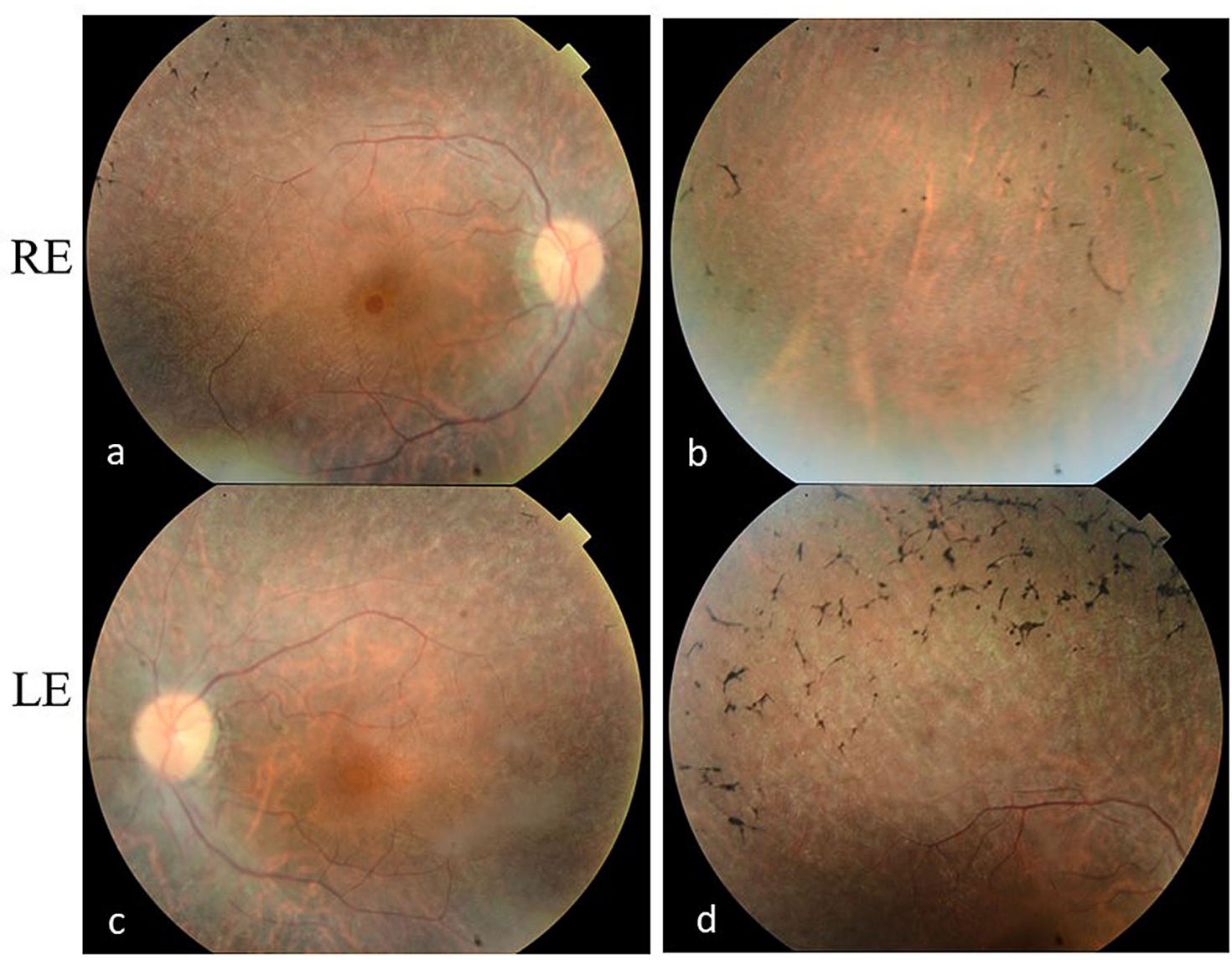

Fig. 1 Pre-operative fundus photographs. a, b Fundus of the right eye showing peripheral retinal atrophy with a few intraretinal pigment deposits in a bone-spicule configuration, retinal vessel attenuation and optic nerve head pallor, while a full-thickness macular hole can be seen in the

ellipsoid zone was only preserved in the fovea (Fig. 2b, d, f).

The patient underwent 23-gauge three-port pars plana vitrectomy (PPV) with internal limiting membrane (ILM) peeling assisted by membrane dual blue and tamponade with $16 \%$ hexafluoroethane $\left(\mathrm{C}_{2} \mathrm{~F}_{6}\right)$ gas. One month after surgery, OCT (RE) confirmed the successful closure of the macular hole with severe disruption of the ellipsoid zone (Fig. 2c). Due to posterior subcapsular cataract development in the $\mathrm{RE}$, the patient subsequently underwent successful uncomplicated cataract surgery with phacoemulsification and intraocular lens implantation 6 months after PPV. At final follow-up, 11 months after PPV, the BCVA macula. $\mathbf{c}, \mathbf{d}$ The left eye also has a typical RP appearance with multiple bone spicules in the periphery and a relatively spared macula. $R E$ right eye, $L E$ left eye

remained stable at 2.5/10 (RE) while the macular hole remained closed and a small part of the ellipsoid zone could be seen (Fig. 2e).

The scotopic full-field electroretinogram (ffERG) was non-recordable in either of the eyes indicating mostly loss of rod system sensitivity (Fig. 3a, b). The responses obtained from the photopic ERG, which entirely assesses cone function, were reduced and delayed in both eyes (Fig. 3c, d). These findings are in keeping with rod-cone dystrophy [1]. Due to the co-existence of severe bilateral sensorineural hearing impairment (data not shown), a clinical diagnosis of Usher syndrome was made.

After written informed consent had been obtained from the patient and family members 


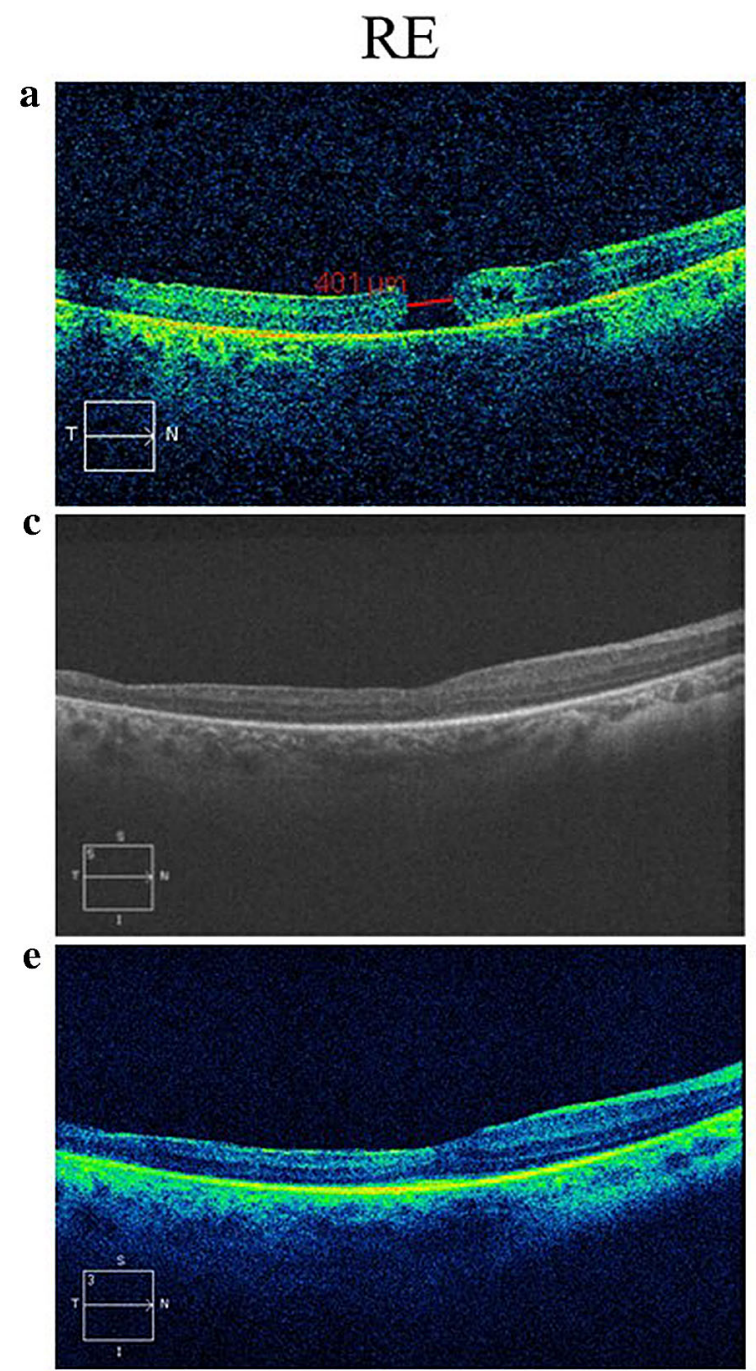

Fig. 2 SD-OCT of the macula of both eyes. a Preoperatively, in the right eye a FTMH (width $401 \mu \mathrm{m}$ ) and a few intraretinal cystic spaces can be seen. There is also thinning of the outer retinal layers and no ellipsoid zone can be seen. $\mathbf{b}, \mathbf{d}, \mathbf{f}$ In the left eye, thinning of the outer retinal layers is observed, while only the foveal part of the ellipsoid zone is preserved. c One month after vitrectomy,

(approved by the Bioethics Committee of the School of Medicine, Aristotle University of Thessaloniki), saliva samples were obtained from the patient and family members for subsequent genomic DNA extraction (Oragene DNA OG-500 saliva kits, DNA Genotek). Genetic testing following whole exome sequencing (WES) analyses revealed that the patient carried two compound heterozygous

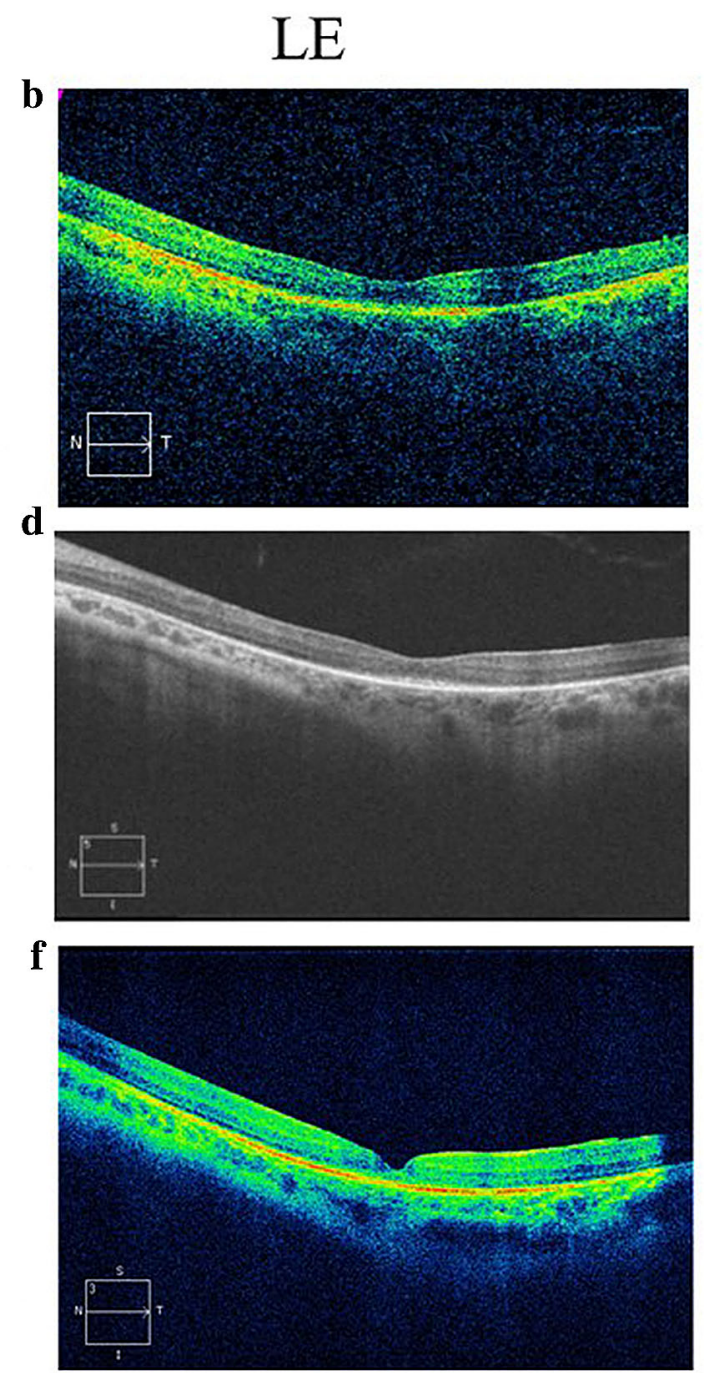

the FTMH is closed in the right eye. e Eleven months after vitrectomy, the macular hole in the right eye remains closed with only few photoreceptors remaining in the temporal part of the fovea. $R E$ right eye, $L E$ left eye

pathogenic variants in the USH2A gene (OMIM 608400, NM_007123.5) and in particular: a nonsense mutation, c.100C $>\mathrm{T}$, p.(Arg34Ter), and a synonymous change c.949C $>$ A, p. $(\operatorname{Arg} 317=)$, which has been shown to lead to a new splice site [9]. The two variants, classified as pathogenic in ClinVar by multiple submitters, segregated with the phenotype in the patient's 
a. DA 0.01 ERG
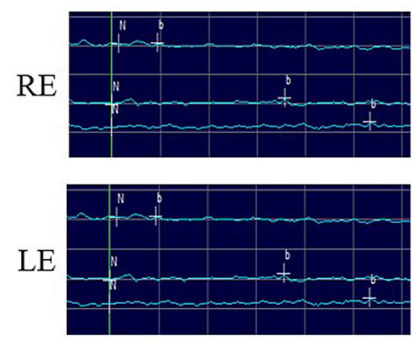

$\mathrm{N}$

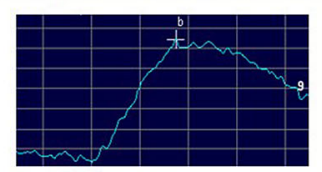

b. DA 10 ERG
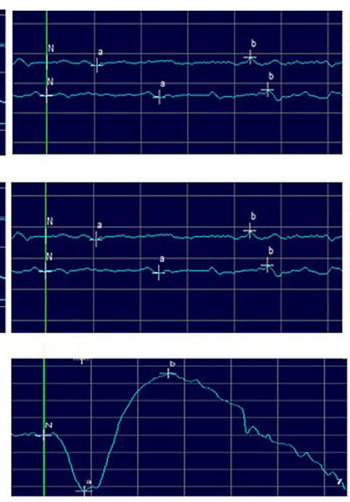

c. LA 3 ERG
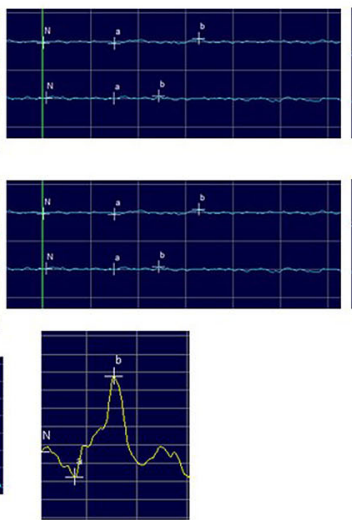

d. LA 30Hz flicker ERG
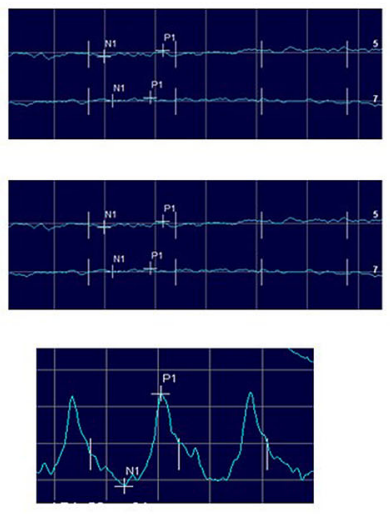

Fig. 3 Full-field ERG findings indicating the presence of rod-cone dystrophy. a, b Under scotopic conditions, the ERG is non-detectable in both eyes. $\mathbf{c}$, $\mathbf{d}$ Under photopic conditions, the responses are reduced and delayed bilaterally. The $\mathrm{x}$ axis represents time in $\mathrm{ms}(20 \mathrm{~ms} / \mathrm{div})$. The

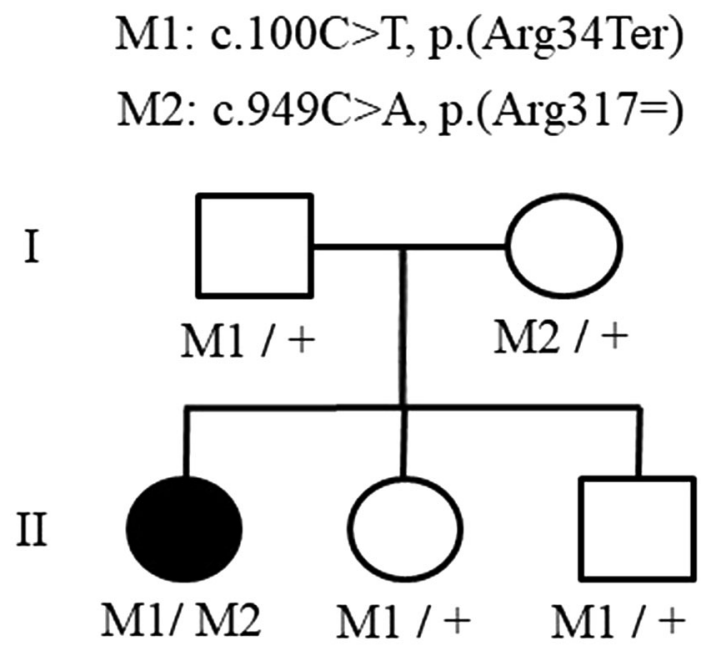

Fig. 4 Genetic analysis of the patient's family showing the pedigree and segregation data for the pathogenic variants in USH $2 A$. The genotypes for all tested family members are shown below each individual, with M1 representing the first mutant allele, c.100C $>$ T, p.(Arg34Ter), M2 the second mutant allele, c.949C $>$ A, p. $($ Arg317=) and + representing the wild-type allele. The affected individual is shaded black

family (Fig. 4). Genetic counselling was given to the patient and family members. $y$ axis represents potential in $\mu \mathrm{V}$ (a, b $50 \mu \mathrm{V} / \mathrm{div}, \mathbf{c}-\mathrm{d}$ $20 \mu \mathrm{V} /$ div). $D A$ dark-adapted, $L A$ light-adapted, $E R G$ electroretinogram, $R E$ right eye, $L E$ left eye, $N$ normal

\section{DISCUSSION}

Macular abnormalities are relatively frequent in $\mathrm{RP}$ patients, the most prevalent being cystoid macular oedema, epiretinal membrane and vitreomacular traction $[7,8,10]$. FTMH in RP has been reported to be rare $(\leq 1 \%)$, although most studies have focused on non-syndromic RP patients [7, 10-12]. The only study including exclusively Usher patients was performed by Testa et al. [13] who demonstrated a high prevalence of macular abnormalities (47\%). In this Italian cohort of 134 genetically confirmed Usher cases, FTMH was found only in one eye of a patient carrying USH2A mutations and thus showing a prevalence of $0.4 \%$ [13].

The mechanism underlying the formation of FTMH in RP remains unclear and is considered multifactorial. Principally, abnormalities of the vitreomacular interface, such as vitreomacular traction and epiretinal membrane, are considered to be a major mechanism [11, 14, 15]. Moreover, atrophy due to chronic cystoid macular oedema and macular schisis in highly myopic eyes could potentially lead to FTMH $[12,15]$. Of note, our patient had posterior vitreous detachment in the affected eye, so our hypothesis is that vitreomacular traction is the underlying mechanism in this case. 
The role of vitrectomy is established in the treatment of idiopathic FTMH leading to a high closure rate and significant VA improvement compared to observation [16]. However, due to the rarity of FTMH cases in the context of RP, surgical outcomes have only been reported in a few small case series including patients with heterogeneous characteristics such as RP stage, symptoms duration, baseline VA, FTMH size and ocular comorbidities [10, 12, 14, 15, 17, 18]. Eight RP cases with FTMH have been reported to have a successful anatomic outcome with seven of them showing higher post-operative VA and one of them having unaltered VA $[10,14,15,18]$. Another four cases with high myopia have been reported, two of which were accompanied by retinal detachment. All four underwent vitrectomy resulting in successful closure, with VA improvement in three patients, while in the fourth one the VA deteriorated $[12,15,18]$. Moreover, in three patients the FTMH failed to close after one vitrectomy and resulted in worse VA $[10,14,18]$. Of note, FTMH reopening after successful surgical intervention has been reported in one patient [17]. Based on these studies, a successful closure of the macular hole is achieved in the majority of the cases, whereas the visual outcomes seem to be mostly positive but can be variable. Thus, the surgical treatment of these cases is usually considered beneficial, notwithstanding the potential risks of vitrectomy, including phototoxicity [19] and visual field defects [20]. The presence of long-standing retinal degeneration may affect the visual outcomes in RP patients. In our patient the FTMH remained closed until the most recent follow-up 11 months post-operatively and the VA remained stable compared to baseline.

Importantly, most of these studies have included patients with either non-syndromic RP or no clear clinical statement (syndromic vs. non-syndromic RP) $[10,12,15,18]$. The only study reporting surgical outcomes of a FTMH case with Usher syndrome was performed by Vingolo et al. [21]. The authors report the results from three cases including two RP patients with lamellar holes and one Usher subject with FTMH who underwent combined microincision vitrectomy and cataract surgery.
The latter case was successful showing a VA improvement from $2 / 20$ to $4 / 20$. However, the underlying mechanism may be different from our report because the macular holes were secondary to "chronic CMO and tangential vitreoretinal tractions". No OCT data were presented for this patient [21].

No genetic data were presented in the aforementioned reports of similar cases. To the best of our knowledge, this is the first case of surgical management of a FTMH in a patient with genetically confirmed Usher syndrome. In our patient, WES revealed two compound heterozygous mutations in USH2A. Pathogenic variants in this gene account for the majority among the entire spectrum of genetic aberrations present in patients with Usher syndrome type 2 [4]. USH2A encodes usherin, localised to the periciliary membrane complex, a region of the photoreceptor inner segment that surrounds the connecting cilium between the outer and inner segment [22]. The stop mutation, p.(Arg34Ter), has been previously reported in several patients with Usher syndrome $[23,24]$. The second pathogenic variant, p.(Arg317=), is predicted to activate a cryptic donor splice site and has also been previously reported $[25,26]$. Its effect has been functionally assessed in vitro at the mRNA level [r.(949C>A, 951_1143del)], where it was shown to lead to the deletion of the last 193 bases of exon 6 and thus result in the introduction of a premature termination codon in exon 7 [9]. We recommend that the genetic defects underlying these patients' RP should be reported, when possible. Apart from the typical benefits of genetic testing, such as obtaining an accurate diagnosis, offering genetic counselling and identifying patients eligible for gene therapy or clinical trials, it could help us establish useful genotype-phenotype correlations, e.g. while examining whether specific genes or mutations are associated with the development of macular hole in RP.

\section{CONCLUSION}

We report a rare case of FTMH in a patient with genetically confirmed Usher syndrome that was 
treated successfully with surgery. Our case report supports the notion that vitrectomy is an effective approach in these cases as demonstrated by the successful anatomical outcome, which stabilised central vision. Surgical management should therefore be selected to try to preserve the central vision of these patients who already may have severely impaired peripheral vision. Further research is needed to enrich our understanding and surgical experience in such cases and potentially identify clinical and/or genetic features related to the development and prognosis of FTMH in the context of RP.

\section{ACKNOWLEDGEMENTS}

Funding. Genetic analyses were supported by the Swiss National Science Foundation (Berne, Switzerland), grant no. 176097, to Carlo Rivolta. No funding or sponsorship was received for the publication of this article.

Authorship. All named authors meet the International Committee of Medical Journal Editors (ICMJE) criteria for authorship for this article, take responsibility for the integrity of the work as a whole, and have given their approval for this version to be published.

Prior Presentation. This manuscript is based on work that was presented at the 15 th Meeting of the Greek Vitreoretinal Society, 30 January-1 February 2020, Athens, Greece.

Disclosures. Evangelia S. Panagiotou, Thomas Papathomas, Konstantinos Nikopoulos, Stavrenia Koukoula, Mathieu Quinodoz, Atta Ur Rehman, Theodoros Giannopoulos and Carlo Rivolta declare that they have no conflict of interest. Anastasios G. Konstas is a member of the journal's Editorial Board.

Compliance with Ethics Guidelines. Written informed consent for the publication of this report and for genetic testing was obtained from the patient and family members (approved by the Bioethics Committee of the School of Medicine, Aristotle University of Thessaloniki).
Data Availability. Data sharing is not applicable to this article as no datasets were generated or analyzed during the current study.

Open Access. This article is licensed under a Creative Commons Attribution-NonCommercial 4.0 International License, which permits any non-commercial use, sharing, adaptation, distribution and reproduction in any medium or format, as long as you give appropriate credit to the original author(s) and the source, provide a link to the Creative Commons licence, and indicate if changes were made. The images or other third party material in this article are included in the article's Creative Commons licence, unless indicated otherwise in a credit line to the material. If material is not included in the article's Creative Commons licence and your intended use is not permitted by statutory regulation or exceeds the permitted use, you will need to obtain permission directly from the copyright holder. To view a copy of this licence, visit http://creativecommons.org/licenses/by$\mathrm{nc} / 4.0 /$.

\section{REFERENCES}

1. Leroy BP. Usher Syndromes. In: Puech B, De Laey J-J, Holder GE, editors. Inherited chorioretinal dystrophies. 1st ed. Berlin: Springer; 2014. p. 143-149.

2. Hope CI. Usher syndrome in the city of Birmingham-prevalence and clinical classification. $\mathrm{Br} \mathrm{J}$ Ophthalmol. 1997;81(1):46-53.

3. Kimberling WJ, Hildebrand MS, Shearer AE, et al. Frequency of Usher syndrome in two pediatric populations: implications for genetic screening of deaf and hard of hearing children. Genet Med. 2010;12(8):512-6.

4. Bocquet B, Lacroux A, Surget MO, et al. Relative frequencies of inherited retinal dystrophies and optic neuropathies in Southern France: assessment of 21-year data management. Ophthalm Epidemiol. 2013;20(1):13-25.

5. Mathur P, Yang J. Usher syndrome: hearing loss, retinal degeneration and associated abnormalities. Biochim Biophys Acta Mol Basis Dis [Internet]. 2015;1852(3):406-20. 
6. RETNET (The Retinal Information Network) [Internet]. https://sph.uth.edu/RetNet/

7. Testa F, Rossi S, Colucci R, et al. Macular abnormalities in Italian patients with retinitis pigmentosa. Br J Ophthalmol. 2014;98(7):946-50.

8. Liew G, Strong S, Bradley P, et al. Prevalence of cystoid macular oedema, epiretinal membrane and cataract in retinitis pigmentosa. Br J Ophthalmol. 2019;103(8):1163-6.

9. Vaché C, Besnard T, Blanchet C, et al. Nasal epithelial cells are a reliable source to study splicing variants in Usher syndrome. Hum Mutat. 2010;31(6):734-41.

10. Hagiwara A, Yamamoto S, Ogata K, et al. Macular abnormalities in patients with retinitis pigmentosa: prevalence on OCT examination and outcomes of vitreoretinal surgery. Acta Ophthalmol. 2011;89(2): 122-5.

11. Fragiotta S, Rossi T, Carnevale C, et al. Vitreomacular interface disorders in retinitis pigmentosa. Graefe's Arch Clin Exp Ophthalmol. 2019;257(10): 2137-46.

12. Liu J, Lyu J, Zhang X, Zhao P. Lamellar hole-associated epiretinal membrane is a common feature of macular holes in retinitis pigmentosa. Eye [Internet]. 2019;34(4):643-9.

13. Testa F, Melillo P, Rossi S, et al. Prevalence of macular abnormalities assessed by optical coherence tomography in patients with Usher syndrome. Ophthalmic Genet [Internet]. 2018;39(1):17-211.

14. Yan F, Xia FJ, Jiang F, Yu HG. Visual and morphological outcomes of vitreomacular traction syndrome in retinitis pigmentosa treated by vitrectomy. Int J Ophthalmol. 2018;11(8):1411-5.

15. Jin ZB, Gan DK, Xu GZ, Nao-I N. Macular hole formation in patients with retinitis pigmentosa and prognosis of pars plana vitrectomy. Retina. 2008;28(4):610-4.

16. Parravano M, Giansanti F, Cm E, Yc Y, Rizzo S, Virgili G. Vitrectomy for idiopathic macular hole. Cochrane Database Syst Rev. 2015;1:5.
17. García-Fernández M, Castro-Navarro J, Bajo-Fuente A. Unilateral recurrent macular hole in a patient with retinitis pigmentosa: a case report. J Med Case Rep. 2013;7:2-5.

18. Ratra D, Raval V. Surgery for macular holes associated with unusual concomitant pathologies. Oman J Ophthalmol. 2013;6(2):112-5.

19. Charles S. Illumination and phototoxicity issues in vitreoretinal surgery. Retina. 2008;28(1):1-4.

20. Yonemura N, Hirata A, Hasumura T, Negi A. Fundus changes corresponding to visual field defects after vitrectomy for macular hole. Ophthalmology. 2001;108(9):1638-43.

21. Vingolo EM, Valente S, Gerace E, Spadea L, Nebbioso M. Macular hole in retinitis pigmentosa patients: microincision vitrectomy with polydimethylsiloxane as possible treatment. Eye. 2015;29(5):699-702.

22. Maerker T, van Wijk E, Overlack N, et al. A novel Usher protein network at the periciliary reloading point between molecular transport machineries in vertebrate photoreceptor cells. Hum Mol Genet. 2008;17(1):71-86.

23. Dreyer B, Tranebjærg L, Rosenberg T, Weston MD, Kimberling WJ, Nilssen $\varnothing$. Identification of novel USH2A mutations: implications for the structure of USH2A protein. Eur J Hum Genet. 2000;8(7):500-6.

24. Bernal S, Medà C, Solans T, et al. Clinical and genetic studies in Spanish patients with Usher syndrome type II: description of new mutations and evidence for a lack of genotype-phenotype correlation. Clin Genet. 2005;68(3):204-14.

25. Pennings RJE, Te Brinke $\mathrm{H}$, Weston $\mathrm{MD}$, et al. USH2A mutation analysis in 70 Dutch families with Usher syndrome type II. Hum Mutat. 2004;24(2): 185.

26. Dreyer B, Brox V, Tranebjaerg L, et al. Spectrum of USH2A mutations in Scandinavian patients with Usher syndrome type II. Hum Mutat. 2008;29(3): 451. 